\title{
PROTECTIVE TREATMENTS FOR LAPPED PORCELAIN STONEWARE TILES AND EVALUATION OF THEIR CLEANABILITY
}

\author{
${ }^{\#}$ ELISA RAMBALDI*, BEATRICE LUCCHESE**, MARIA CHIARA BIGNOZZI*,** \\ *Centro Ceramico, via Martelli 26, Bologna, Italy \\ **Department of Civil, Chemical, Environmental and Materials Engineering, University of Bologna, \\ via Terracini 28, Bologna, Italy \\ "E-mail: rambaldi@centroceramico.it
}

Submitted December 22; accepted March 13, 2017

\begin{abstract}
Keywords: Porcelain stoneware tiles, Lapping, Stain proofing agent, Dirt resistance
Since the arrival of lapped porcelain stoneware tiles on the market, several studies have been focused on the improvement of the technical characteristics of the surfaces of these products. Surface lapping induces aesthetical improvements, but can at the same time deteriorate the performance of porcelain stoneware tiles. To overcome this problem, it is possible to protect the lapped surface with commercial waterproofing materials. In this work, lapped commercial porcelain stoneware tiles with protective stain proofing agents (FILA PD15 and FILA 1239 Plus) were evaluated. The stain resistance and chemical resistance results were correlated to the morphological surface characteristics of the products with and without protection. A systematic study of the surface porosity of the tiles was carried out. Results showed that unprotected surface pores tend to fill with dirt that is hardly removable by ordinary maintenance. If the pores are protected, the dirt from foot traffic is deposited only superficially and can be removed.
\end{abstract}

\section{INTRODUCTION}

The main type of ceramic tiles is porcelain stoneware, an extremely vitrified material characterized by a very compact structure and excellent technical performance as a result of high density (water absorption $<0.5 \%$ ), abrasion and stain resistance, and surface hardness. Other types of stoneware, such as singlefired tiles, like so-called monoporosa, double-fired or clinker tiles, are more porous with a water absorption $<5 \%$ according to EN 14411 [1]. During firing, porcelain stoneware tiles are exposed to relatively high temperatures, up to $1250^{\circ} \mathrm{C}$, which allows a very durable and compact surface to be achieved. However, in order to attain aesthetic characteristics that are highly valued by the end user, stoneware tiles are often lapped to reduce their surface roughness and to increase their gloss [2].Therefore lapped porcelain stoneware is nowadays the most appreciated ceramic product on the market, representing about $20 \%$ of the whole Italian production of porcelain stoneware tiles [3].

Although the lapping process improves the aesthetic appearance of the product and increases its competitiveness with natural stone, it also induces several changes on the surface microstructure, as in the case of polishing. A fine layer of product $(<1 \mathrm{~mm})$ is removed, causing the formation of surface cracks and flaws (grooves, scratches) and exposing the closed porosity, which is initially located inside the bulk material, to the surface.
This may compromise the technical performance and aesthetic appearance of stoneware tiles, particularly in terms of cleanability and stain resistance [4-8]: in fact, dirt and staining agents may penetrate into pores, grooves and scratches, and cleaning can be very difficult.

In order to achieve a high stain resistance and to preserve the aesthetic appearance of porcelain stoneware, protective stain proofing agents can be applied on the surface of the tiles. The role of the protective treatment is to fill up open pores and surface cracks, thus preventing the penetration of dirt and staining agents. The effect of protective coatings has been analyzed in previous works [9-11] and it has been demonstrated that the efficiency of the treatments depends on the characteristics of the treatment and on the characteristics of the surface to which it is applied. In this work the effect of two protective treatments on the stain resistance and chemical resistance of commercial lapped porcelain stoneware tiles is studied, the first being applied directly in the production line, the second after tiling.

\section{EXPERIMENTAL}

One type of commercial lapped porcelain stoneware tile $(60 \times 30 \mathrm{~cm}$; white color $)$, named "A", was selected for this study. The product belongs to group BIa according to EN 14411 [1], being characterized by values of water absorption lower than 0.5 wt. \%. 
The quantitative mineralogical composition of the fired tile was determined by X-ray diffraction analysis (PW3830, Philips). The powdered specimen, diluted with 10 wt. \% of corundum NIST 676 as an internal standard, was side loaded to minimize preferred orientation. Data were collected in the angular range $10-80^{\circ} 2 \vartheta$ with steps of $0.02^{\circ}$ and $5 \mathrm{~s} / \mathrm{step}$ and the Rietveld refinement was performed using GSAS-EXPGUI software. Results are reported in Table 1.

Table 1. Quantitative mineralogical composition of the fired tile "A".

\begin{tabular}{lc}
\hline Quartz, wt. \% & $19.0 \pm 0.2$ \\
Mullite, wt. \% & $6.8 \pm 0.4$ \\
Zircon, wt. \% & $4.6 \pm 0.2$ \\
Plagioclase, wt. \% & $5.6 \pm 0.3$ \\
Amorphous phase, wt. \% & $64 \pm 1$ \\
\hline
\end{tabular}

This type of lapped tiles was subjected to different treatment steps named as follows:

- A1 - untreated tile (as a reference).

- A2 - tile washed with a phosphoric acid based solution (10 wt. \%) to remove residuals of the lapping process; this treatment was performed during the industrial production process.

- A3 - tile washed with a phosphoric acid based solution and then treated with a protective agent (FT1239 Plus, a treatment produced by FILA and based on nanometric colloidal particles in suspension); this treatment was also performed during the industrial production process.

- A4 - tile washed with a phosphoric acid based solution and treated with two protective agents (FT1239 Plus and, subsequently, FILA PD 15, a mixture of siloxanic compounds dispersed in dearomatised hydrocarbon solvent produced by FILA); the application of FILA PD 15 was performed by the producer by uniformly brushing and removing the excess with a microfiber cloth.

The working surfaces of the tiles were subjected to water contact angle determination. It was performed according to the sessile drop method, an optical technique to determine the wettability of a solid surface, according to the European standard EN 15802 [12]. A stain resistance test, according to international standard ISO 10545-14 [13] and a chemical resistance test, according to ISO 10545-13 [14] (glazed tile procedure) were also performed, together with a surface microstructural analysis. For the stain resistance test, the staining agents were: a green staining agent in light oil $\left(\mathrm{Cr}_{2} \mathrm{O}_{2}\right)$, iodine (13 $\mathrm{g} \cdot \mathrm{l}^{-1}$ solution in alcohol), and olive oil. For the chemical resistance test the solutions were: ammonium chloride solution $\left(100 \mathrm{~g} \cdot \mathrm{l}^{-1}\right)$, sodium hypochlorite solution $\left(20 \mathrm{mg} \cdot \mathrm{l}^{-1}\right)$, hydrochloric acid solution (3 vol. \%), and potassium hydroxide solution $\left(30 \mathrm{~g} \cdot \mathrm{l}^{-1}\right)$. Both tests were performed on 3 different areas of each tile.
An optical profiler (Leica DCM 3D) was used to determine the surface roughness of the samples (ISO 4287 [15], by using a cut-off of $0.8 \mathrm{~mm}$ ), in particular the average roughness, $\mathrm{Ra}$, and the maximum height of the profile, Rz. At least 10 profiles (12 $\mathrm{mm}$ length) were collected for each sample, according to EN 623-4 [16].

An optical microscope (Leica DMLM) was used to analyze the effect of the protective treatment on the working surface of the porcelain stoneware. The reflected light digital images were analyzed using image software (Leica Application Suite/LAS) to calculate the percentage of the total porosity (as a mean value of 5 images for each sample) and to evaluate the pore size distribution by measuring the area equivalent circle diameters (calibration: 1 pixel corresponds to $0.33 \mathrm{~mm}$ ).

Each tile was also examined by scanning electron microscopy / SEM (Zeiss EVO 40) in order to investigate in greater detail the effect of the protective treatment on the surface microstructure of porcelain stoneware. Further correlation between microstructure and cleanability was evaluated by spraying an aqueous suspension of soiling agents and, after drying, by removing it by a dry and a wet cloth. This soiling mixture was prepared on the basis of air pollution data of ARPA, a regional agency of the Bologna area (Italy). It is characterized by a $\mathrm{pH}$, being composed of carbon black $\left(0.25 \mathrm{~g} \cdot \mathrm{l}^{-1}\right)$, an organic compound (uric acid, $1.4 \mathrm{~g} \cdot \mathrm{l}^{-1}$ ), mineral powders (iron oxide and clay, $\left.2.3 \mathrm{~g} \cdot \mathrm{l}^{-1}\right)$ and salts $\left(\mathrm{NaCl}, \mathrm{KNO}_{3}, \mathrm{CaSO}_{4}\right.$, $\left.1 \mathrm{~g} \cdot \mathrm{l}^{-1}\right)$.

\section{RESULTS AND DISCUSSION}

Figure 1 shows that the water contact angle varies significantly between the surfaces A1, A2, A3 and A4. Quantitative results are reported in Figure 1a, from which it is clear that samples A1 and A2 show an intermediate behavior $\left(50-60^{\circ} \theta\right)$, while sample A3 is closer to hydrophilic and sample A4 is closer to hydrophobic behavior. In Figure $1 \mathrm{~b}$ the extremely different wetting behavior of surfaces A3 and A4 with water is shown.

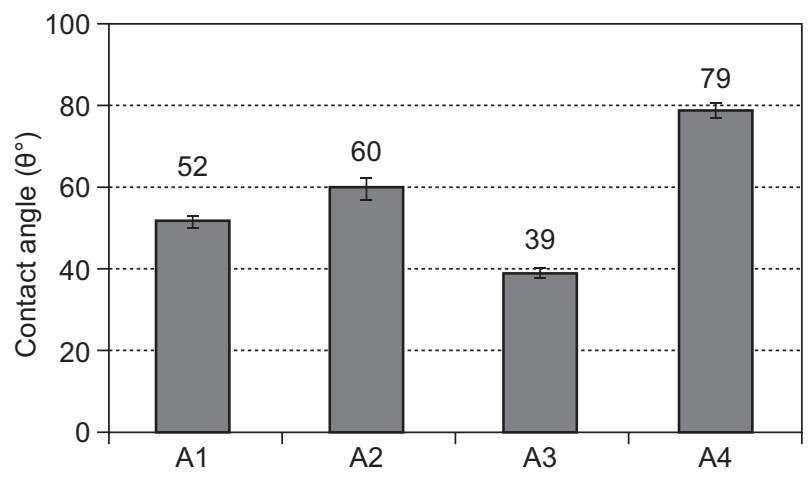

a)

Figure 1. Water contact angle values (a); images of A3 and A4 surfaces wetted with water (b). 

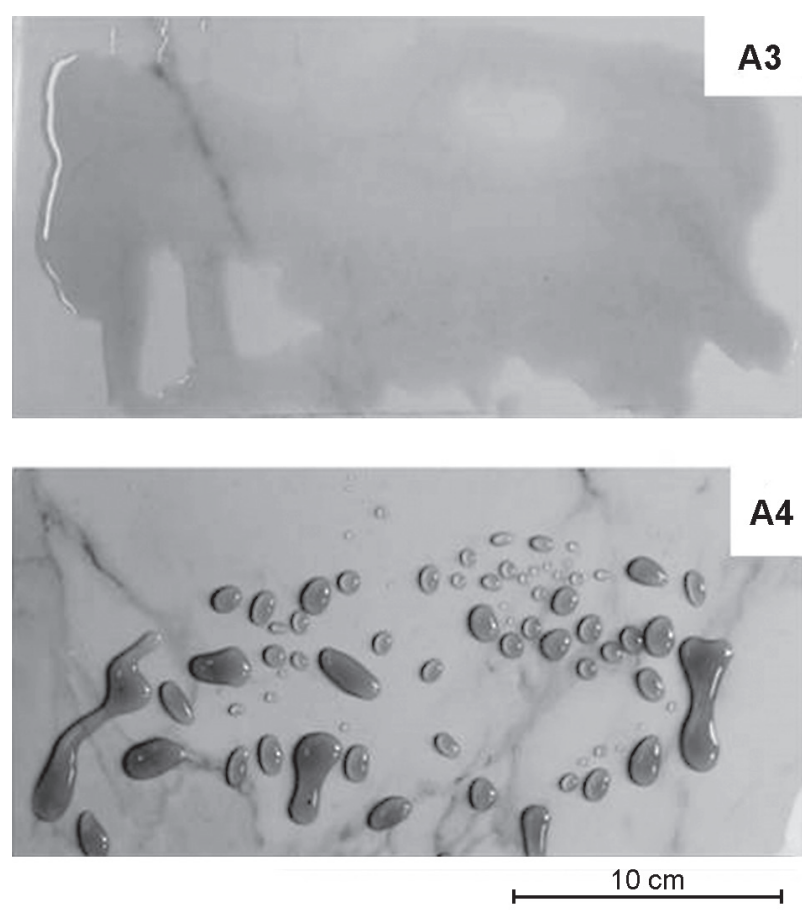

b)

Figure 1. Water contact angle values (a); images of A3 and A4 surfaces wetted with water (b).

Table 2. Results of stain tests (according to ISO 105454-14 classified from 1 to 5 ; class 1 - stain not removed, 2 - stain removed by dipping in a suitable solvent for 24 hours, 3 - stain removed by mechanical cleaning and strong cleansing agent, 4 - stain removed by manual cleaning with weak cleansing agent, 5 - stain removed by means of hot current water for 5 minutes).

\begin{tabular}{cccc}
\hline Samples & $\begin{array}{c}\text { Green staining agent } \\
\text { in light oil }\end{array}$ & Iodine & Olive oil \\
\hline A1 & 5 & 5 & 5 \\
A2 & 5 & 5 & 5 \\
A3 & 5 & 5 & 5 \\
A4 & 5 & 5 & 5 \\
\hline
\end{tabular}

Results of the stain resistance tests (ISO 10545-14) are reported in Table 2. According to this ISO standard all the samples belong to class 5 (i.e. the stain is removed by hot water) and no differences are observed among them, at least for the selected staining agents.

Results of the determination of the chemical resistance (ISO 10545-13) are reported in Table 3. A deterioration of the chemical resistance by using household chemicals (ammonium chloride and sodium hypochlorite solutions) was observed for sample A2.

When using hydrochloric acid solution (low concentration), only a slight deterioration of chemical resistance (class LB) was observed for samples with a protective treatment (A3 and A4), while for tiles with no treatment (A1 and $\mathrm{A} 2$ ) the deterioration is more pronounced (class LC). When using potassium hydroxide solution (low concentration), a marked deterioration of the chemical resistance was observed for all samples.

The roughness determination results are reported in Figure 2. Considering the standard deviation, the average roughness $\mathrm{Ra}$ is very similar in samples $\mathrm{A} 1$, A2 and A3 with or without the protective treatments, being lower only in sample A4. Also the maximum roughness $\mathrm{Rz}$ is considerably lower in sample A4. Nevertheless, these changes do not significantly affect the macroscopic characteristics of the surfaces, e.g. in terms of slipperiness. All roughness values are typical of smooth surfaces such as lapped tiles.

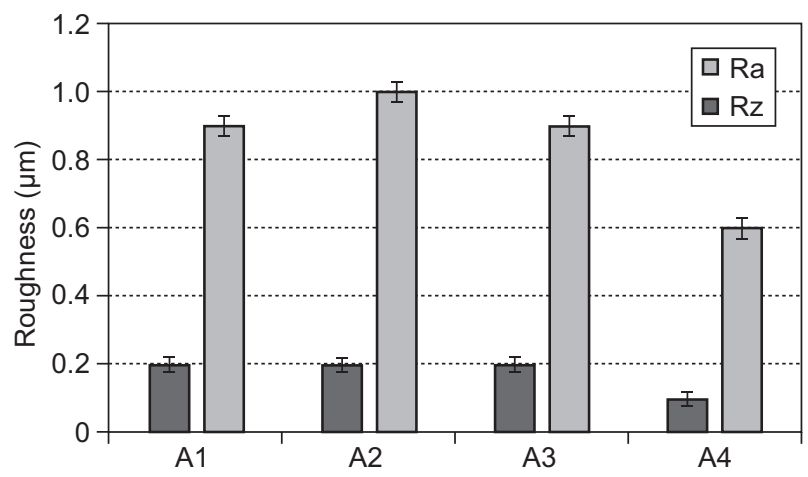

Figure 2. 2D roughness parameters Ra (average roughness) and $\mathrm{Rz}$ (maximum height of the profile).

Table 3. Results of chemical resistance tests when using common household chemicals, ammonium chloride and sodium hypoclorite solutions (according to ISO 105454-14 classified from A to C; class A - no visible effect, class B - discernable visible change in appearance, class $\mathrm{C}$ - partial or complete loss of the original surface) and when using low-concentration acids and alkaline solutions, hydrochloric acid and potassium hydroxide solution (class LA - no visible effect, class LB - discernable change in appearance, class LC - partial or complete loss of the original surface).

\begin{tabular}{ccccccc}
\hline Samples & $\begin{array}{c}\text { Ammonium } \\
\text { chloride }\end{array}$ & $\begin{array}{c}\text { Sodium } \\
\text { hypochlorite }\end{array}$ & $\begin{array}{c}\text { Hydrochloric } \\
\text { acid }\end{array}$ & $\begin{array}{c}\text { Potassium } \\
\text { hydroxide }\end{array}$ & $\begin{array}{c}\text { Pencil } \\
\text { test }\end{array}$ & $\begin{array}{c}\text { Reflection } \\
\text { test }\end{array}$ \\
\hline A1 & A & A & LC & LC & No & Yes \\
A2 & B & B & LC & LC & No & Yes \\
A3 & A & A & LB & LC & Yes & Yes \\
A4 & A & A & LB & LC & Yes & Yes \\
\hline
\end{tabular}



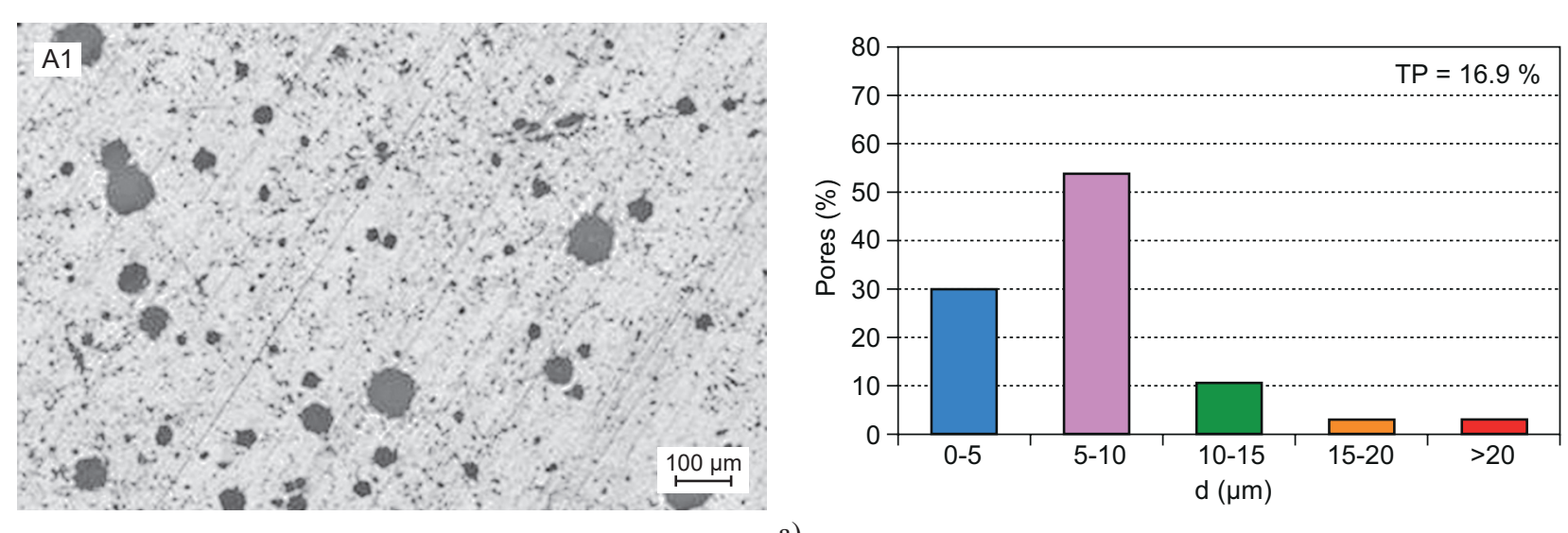

a)
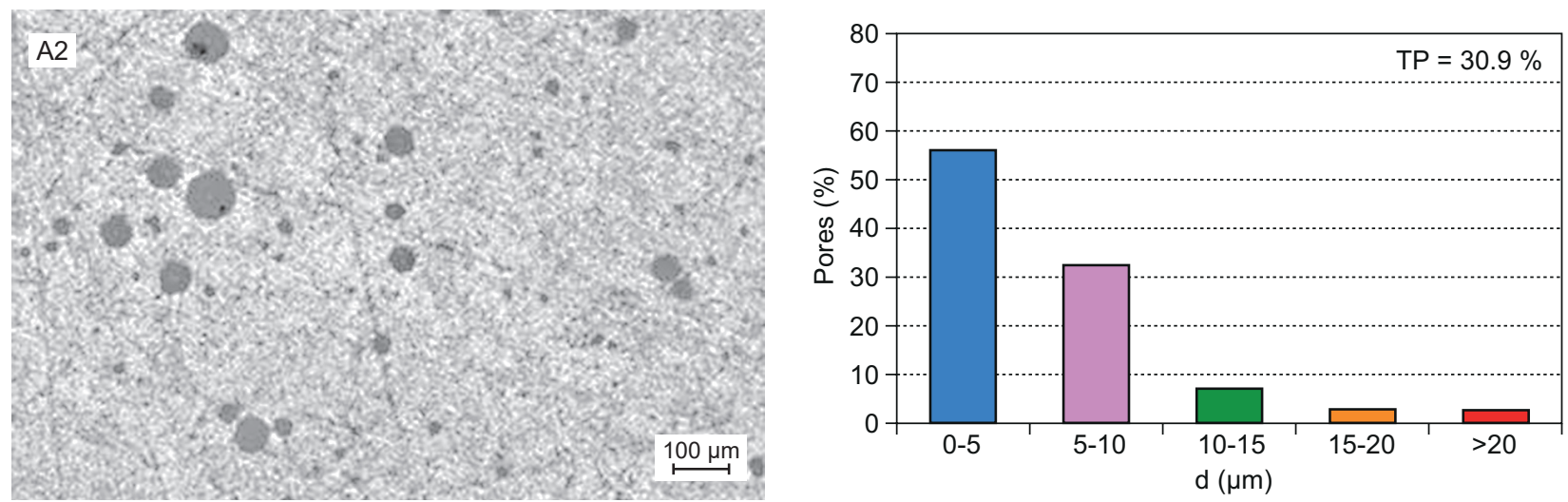

b)
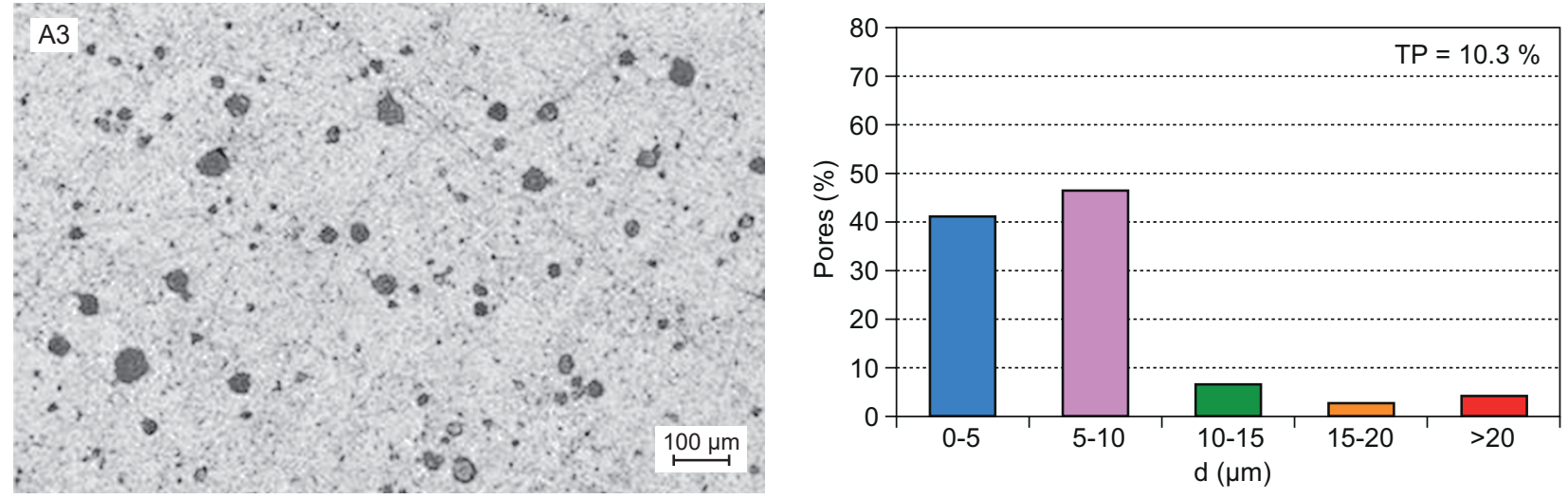

c)
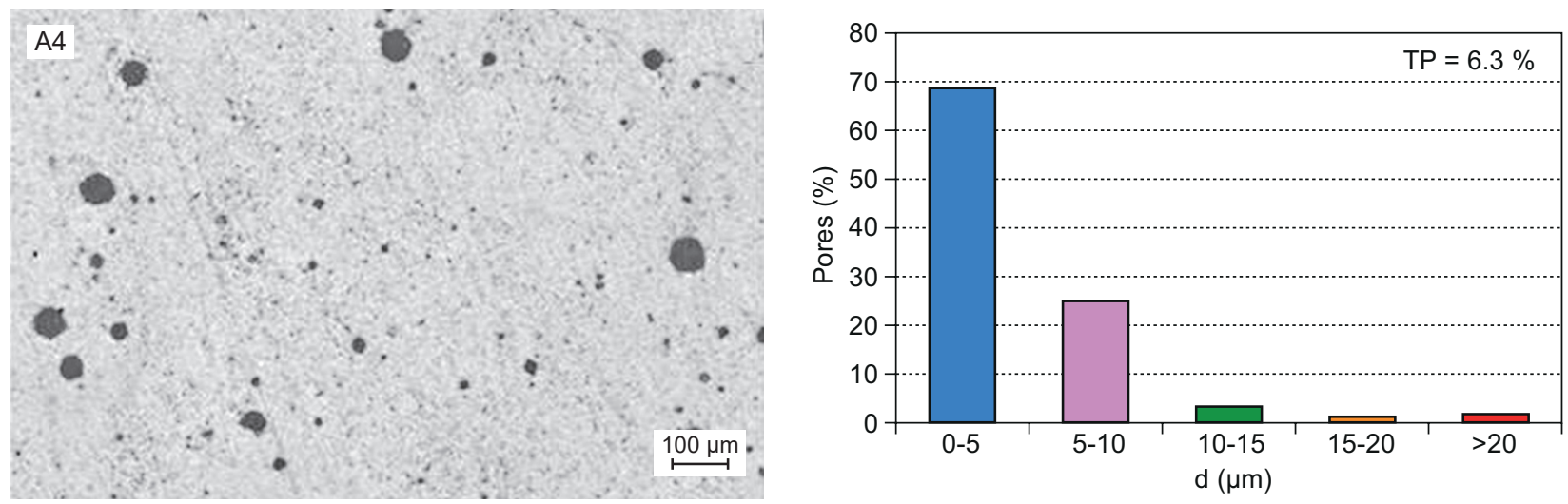

d)

Figure 3. Optical microscope images, total porosity (TP) and pore size distribution for samples A1 (a), A2 (b), A3 (c) and A4 (d). 


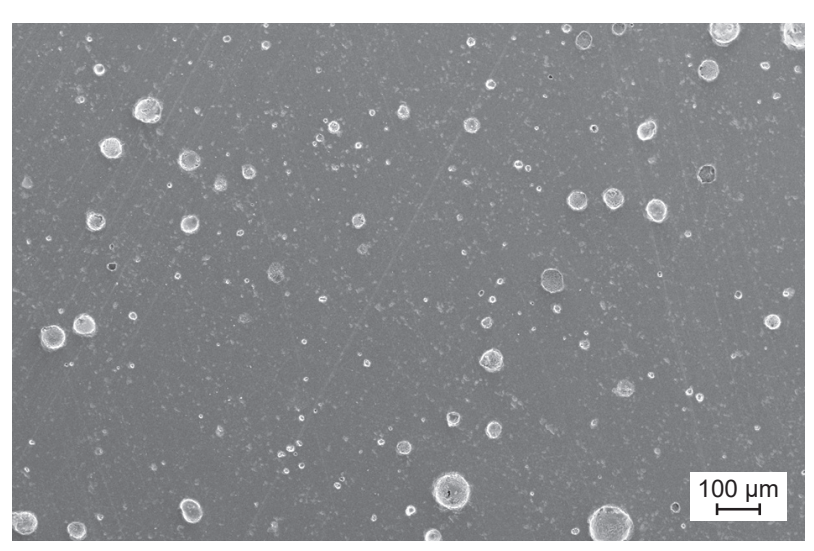

a)

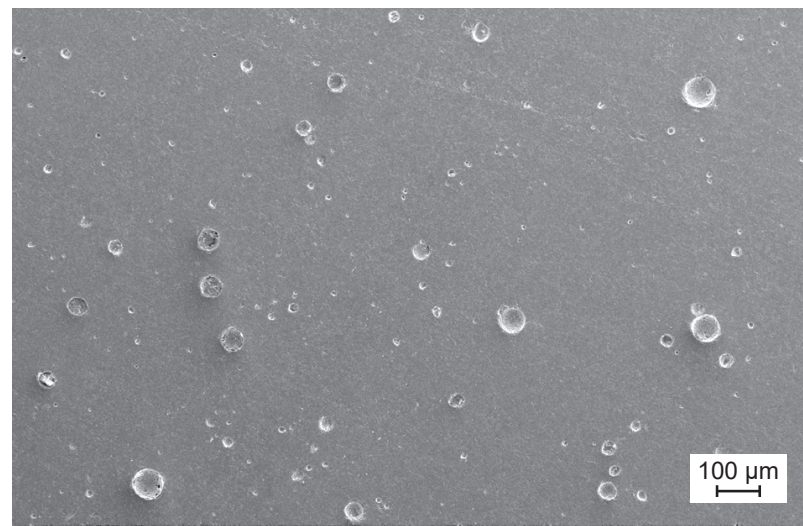

c)

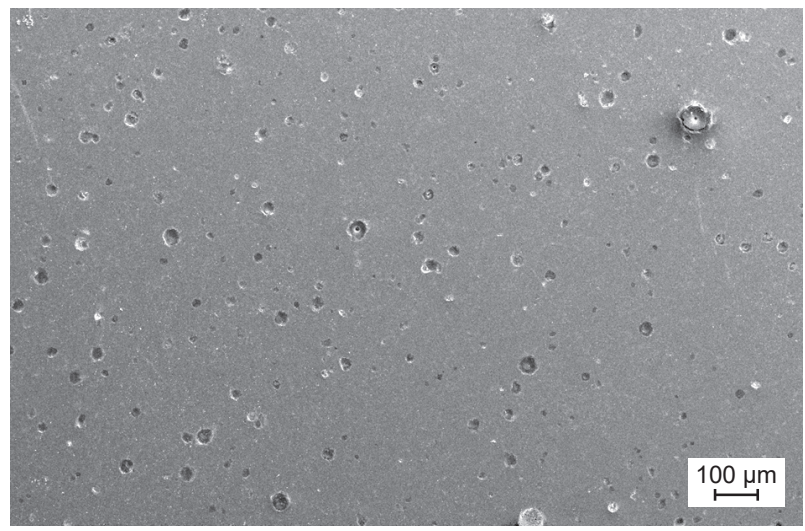

e)

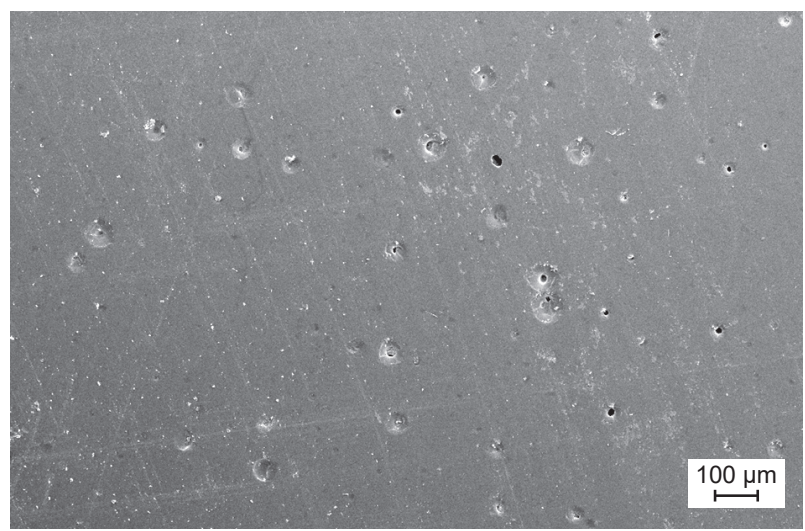

g)

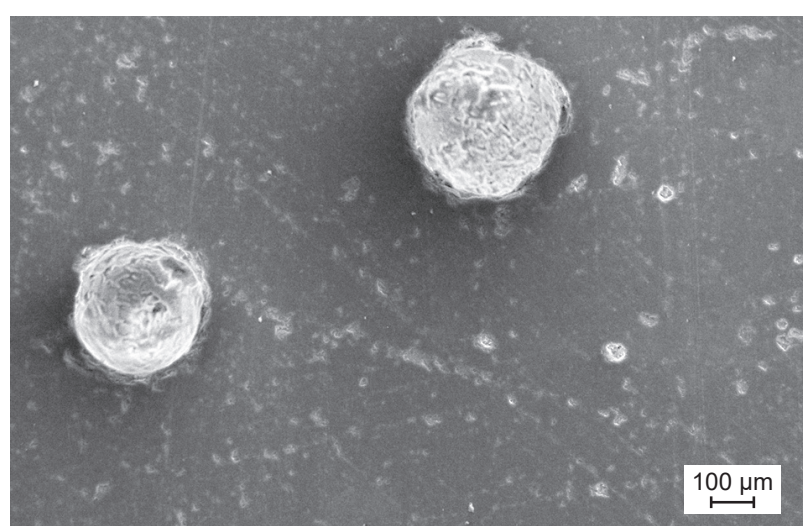

b)

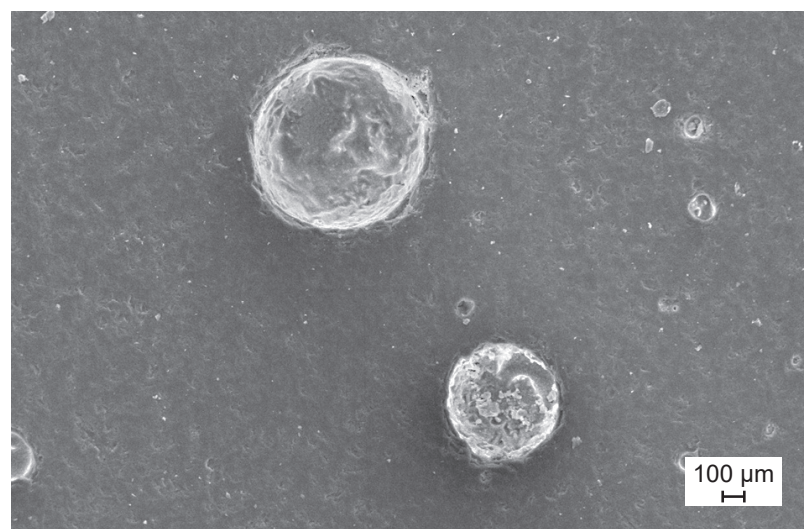

d)

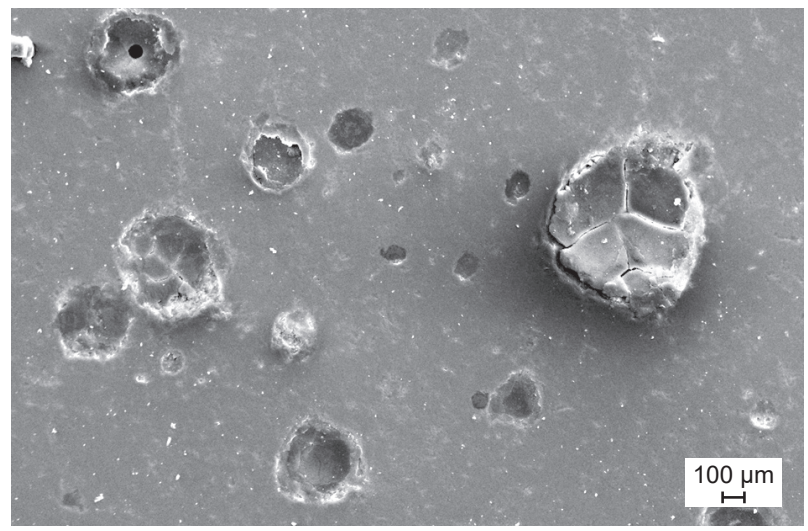

f)

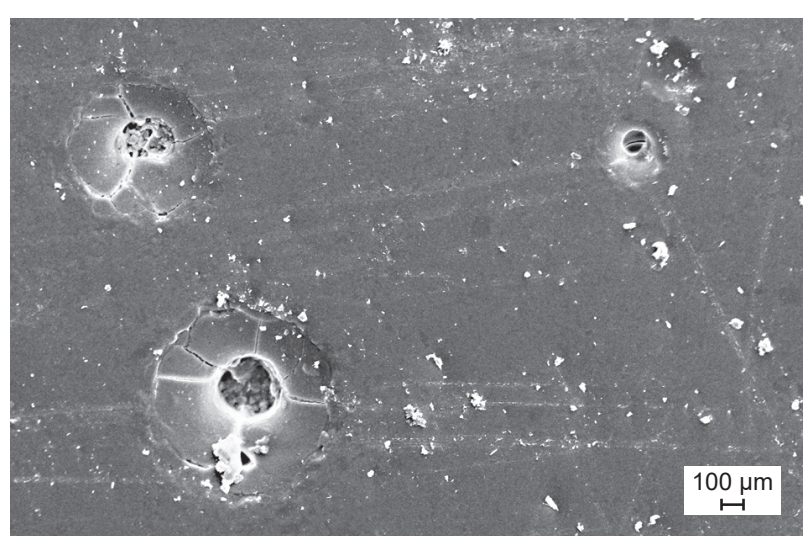

h)

Figure 4. SEM images of sample A1 (a,b), A2 (c,d), A3 (e,f) and A4 (g,h). 
The results of optical microscopy and image analysis are shown in Figure 3 in which the total porosity values (TP) are reported for each samples. It should be emphasized that the TP value represents the percentage of open holes accessible from the surface of the tile surfaces and not filled by the protective agents, thus it does not represent the porosity of the material itself. For sample A1 the value of TP is quite high, $16.9 \%$. The majority of the pores belong to the size classes $5-10 \mu \mathrm{m}$ and $0-5 \mu \mathrm{m}$. Pores measuring $>10 \mu \mathrm{m}$ represent, in total, only about $16 \%$. Apart from pores proper, also grooves and scratches are present that may affect the total porosity value determined via image analysis. For sample A2 (washed with phosphoric acid solution) the total porosity increases significantly, reaching a value of $30.9 \%$, due to the effect of the acid that dissolves the glassy phase and creates, in addition to the intrinsic porosity, a large amount of small irregular cavities having sizes below $5 \mu \mathrm{m}$. For sample A3 (treated with the proofing agent FT1239 Plus) the total porosity decreases to a value of $10.3 \%$. A great part of the pores below $5 \mu \mathrm{m}$, observed in sample A2, is filled and covered by the treatment. However, pores measuring more than $10 \mu \mathrm{m}$ are only partially filled (this effect is more clearly visible in SEM images, Figure 4).

For sample A4 the effect of the treatment is even more evident compared to sample A3. The total porosity decreases to a value of $6.3 \%$. The most part of pores $5-10 \mu \mathrm{m}$ is filled and covered by protective agents, only pores measuring larger than 10 or $20 \mu \mathrm{m}$ are not completely filled, contributing to increase the porosity 0 - $5 \mu \mathrm{m}$ (also this effect can be more easily evaluated by SEM images).

The SEM investigation of sample A1 (Figure 4a, b) shows more clearly the shape of the pores: pores larger than $10 \mu \mathrm{m}$ have an essentially circular shape, while pores smaller than $10 \mu \mathrm{m}$ are more irregular in shape and are often the result of the coalescence of small pores or of the coalescence of small irregular cavities formed due to the removal of material by the lapping machine. Several marks and scratches impressed by the lapping process are evident on the surface of the sample as well.

The SEM investigation of sample A2 (Figure 4c, d) shows the effect of the phosphoric acid solution on the working surface of the tile. The acid solution dissolves the glassy phase of porcelain stoneware, creating a rough surface. Many irregular hollow spaces, together with the intrinsic porosity of the product, can be observed on the surface of this sample.

The SEM investigation of the sample A3 (Figure $4 \mathrm{e}, \mathrm{f}$ ) shows that the proofing agent covers small pores, hollow spaces created by the acid solution and fills, almost completely, pores larger than $10 \mu \mathrm{m}$. For sample A4 (Figure 4g, h), the effect of the treatment is even more

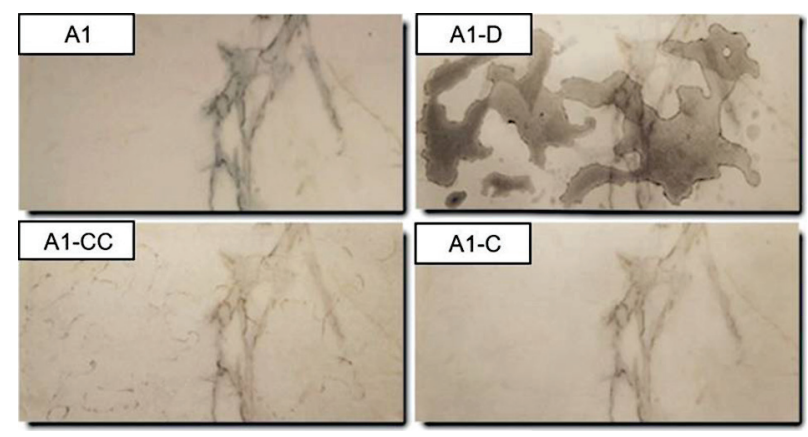

a)

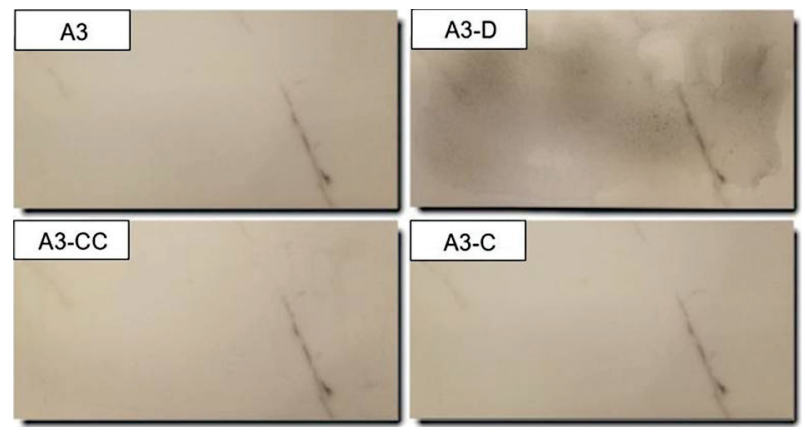

c)
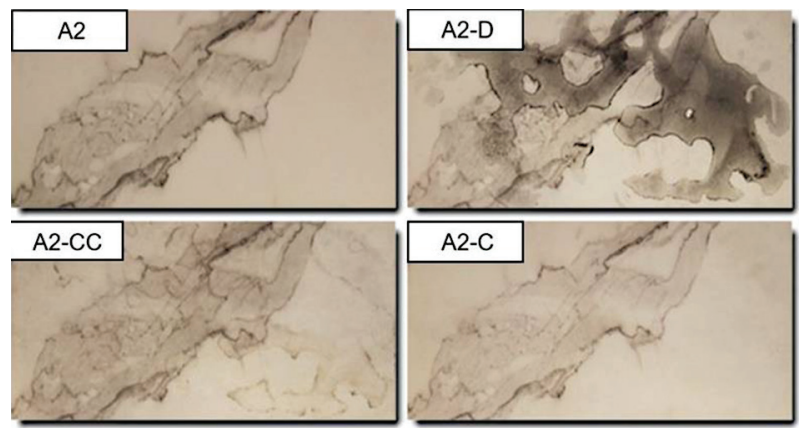

b)

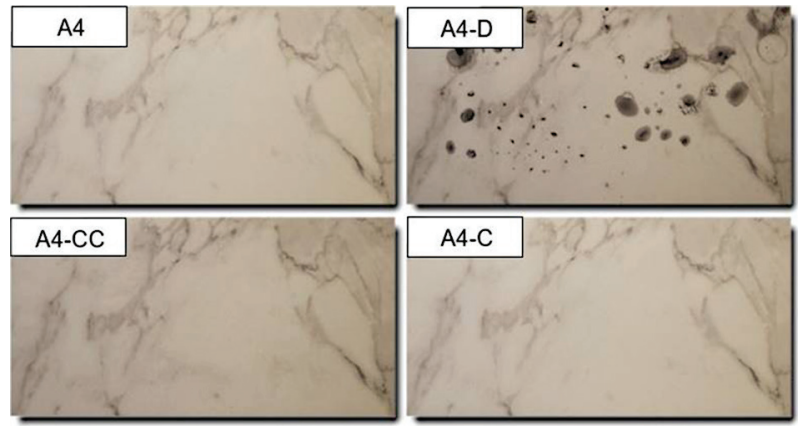

d)

Figure 5. Images (macroscopic photographs) of the tile samples (dimensions $15 \times 30 \mathrm{~cm}$ ) for the cleanability test for sample A1 (a), A2 (b), A3 (c) and A4 (d). For each sample the original clean surfaces (labeled A1, A2, A3 and A4), the surfaces after 4 repeated applications of the "dirt suspension" (A1-D, A2-D, A3-D, A4-D), the surfaces after cleaning with a dry cloth (A1-CC, A2-CC, A3-CC, A4-CC) and with a wet cloth (A1-C, A2-C, A3-C, A4-C) are reported. On the surfaces A3-C and A4-C the dirt is completely removed, on the surfaces $\mathrm{A} 1-\mathrm{C}$ and $\mathrm{A} 2-\mathrm{C}$ only partially. 


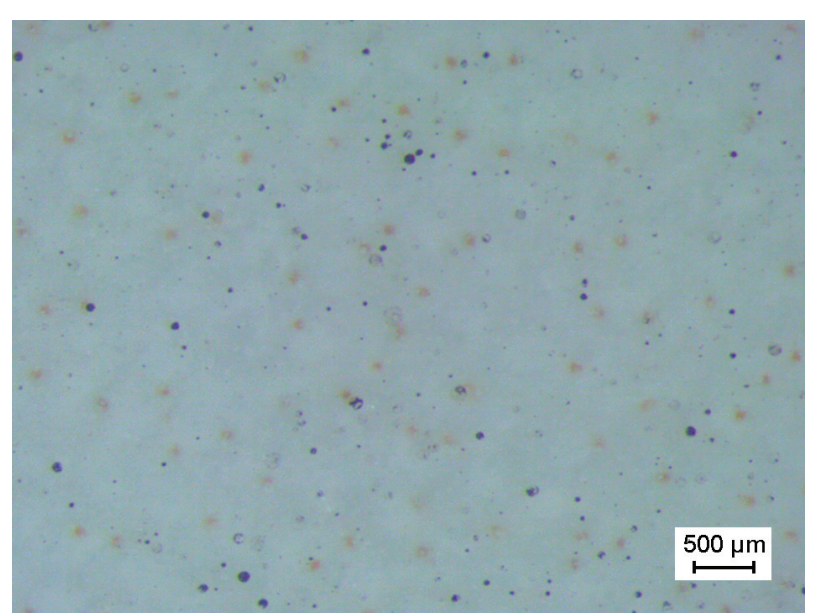

a)

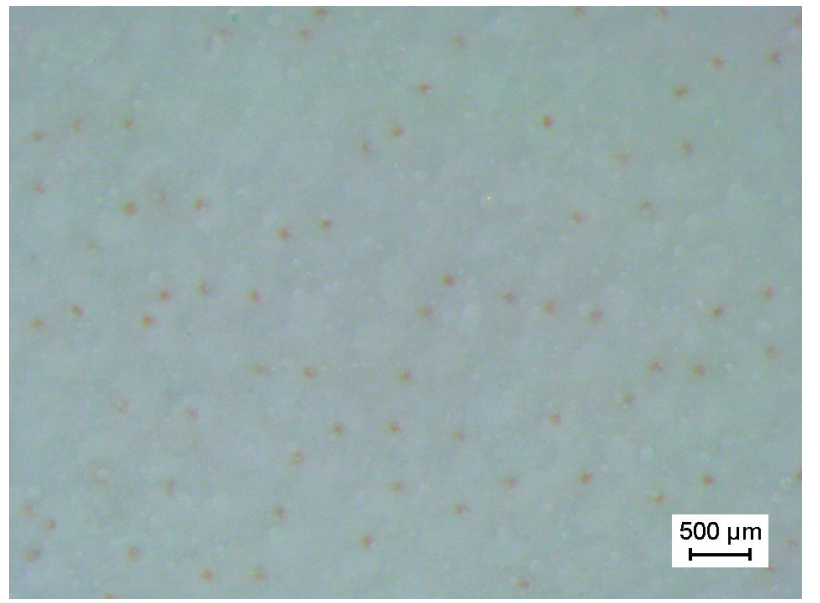

c)

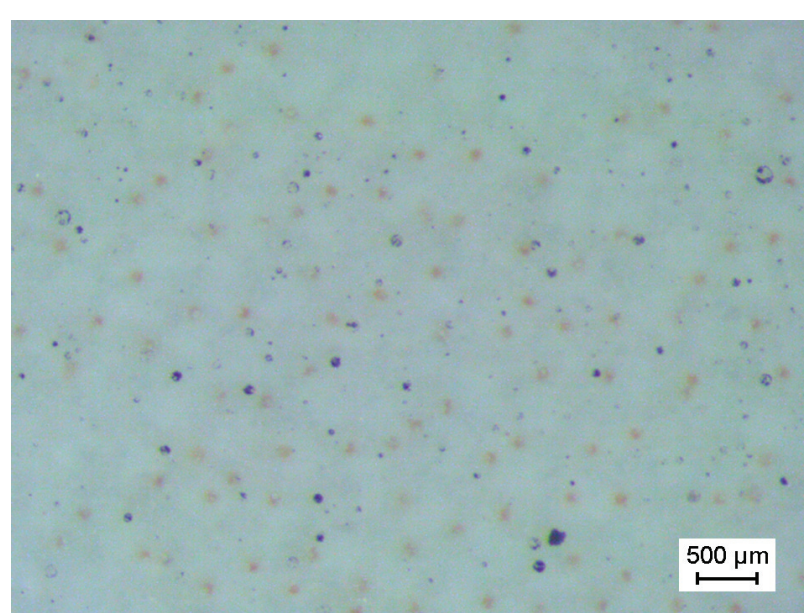

b)

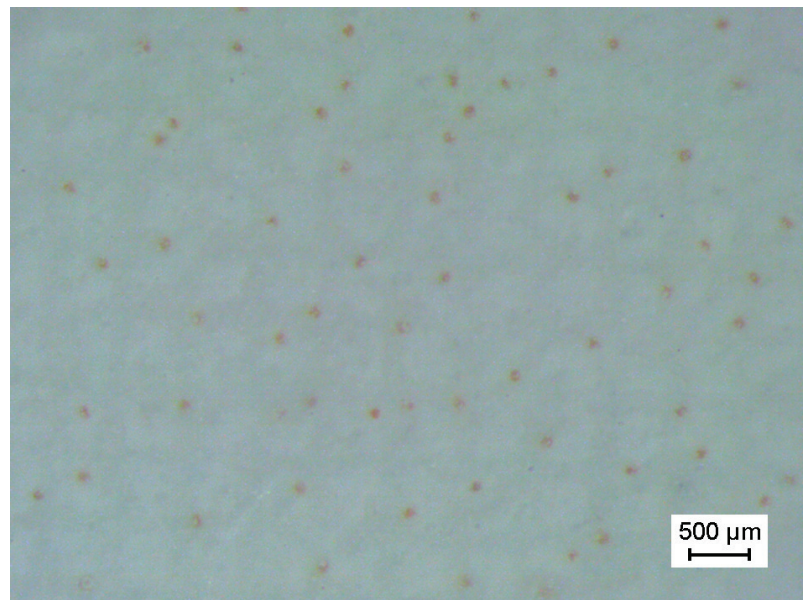

d)

Figure 6. Optical microscope images of the samples surface A1-C (a), A2-C (b), A3-C (c), A4-C (d) after cleaning with a wet cloth.

evident. Only pores larger than $10 \mu \mathrm{m}$ are well visible. These pores are almost totally filled by the proofing agent that appears cracked and, often, leaves a small circular hole in the middle of the pore, contributing to increase the porosity range $0-5 \mu \mathrm{m}$ as observed in Figure 3 .

The cleanability test was carried out by using an aqueous suspension of soiling agents applied on the sample surface. About $25 \mathrm{~g}$ of the soiling mixture was applied by airbrush. After drying at room temperature for 24 hours, the surfaces were cleaned with a dry cloth and then with a wet cloth. This procedure was repeated 4 times on the same surfaces, and the results are shown in Figure 5. On samples A1 and A2 (non-protected surfaces) the dirt cannot be easily removed. Visual inspection of the surfaces after cleaning and comparison with the original clean surfaces, reveals some stains and holes that are visible both by cleaning with a dry (A1$\mathrm{CC}$ and $\mathrm{A} 2-\mathrm{CC})$ or with a wet cloth (A1-C and A2-C). On the samples with one (A3) or two (A4) protective agent treatments, it seems from visual inspection that the cleaning procedure with wet cloth (A3-C and A4-C) is sufficient to remove the dirt completely.

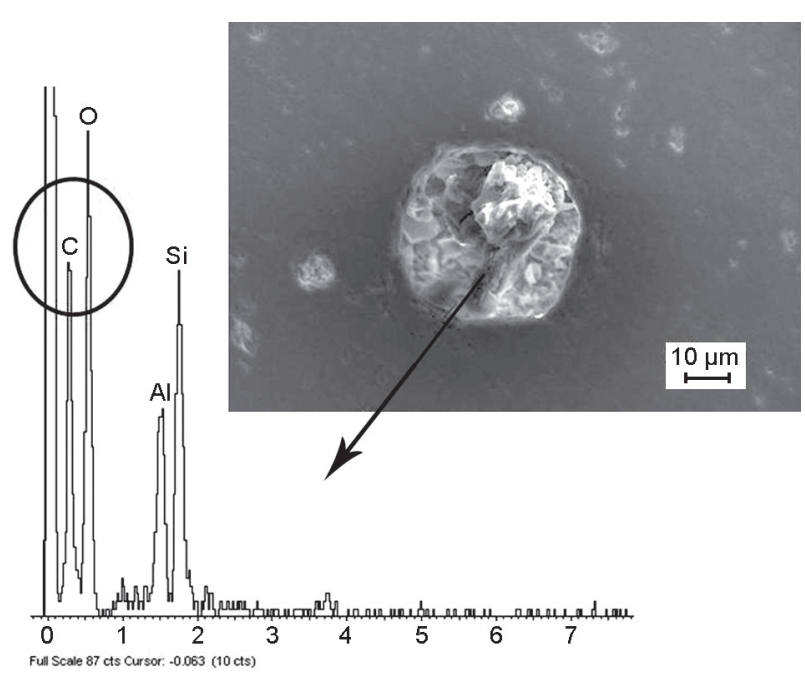

Figure 7. SEM micrograph and EDS microanalyses of a pore in A1, after cleaning, reveal the presence of carbon from the "dirt suspension". 
These results are confirmed by observing the surfaces by an optical microscope (Figure 6). The micrographs of samples A1-C and A2-C after cleaning with a wet cloth, show some pores that are filled with particles coming from the aqueous suspension of soiling agents, as confirmed also by SEM-EDS analysis (Figure 7). The optical micrographs of the samples A3 and A4 do not show the presence of filled pores.

\section{CONCLUSIONS}

Protective treatments on lapped porcelain stoneware tiles do not change the aesthetical appearance of the porcelain stoneware surfaces. In terms of cleanability, the internationally standardized tests do not reveal any significant differences among the ceramic surfaces, protected or not. On the other hand, laboratory tests show important differences due to the different microstructure of the investigated surfaces. Cleanability of ceramic tiles (easy removal of stains, dirt and powder and complete recovery of the original aspect) is an important characteristic that has to be considered to promote and boost a product that, during its lifetime, should guarantee both aesthetical quality and technical performance.

\section{Acknowledgement}

This work was carried out in collaboration of Fila Industria Chimica Spa, S. Martino di Lupari, Padova, Italy.

\section{REFERENCES}

1. EN 14411: 2016. Ceramic tiles - Definition, classification, characteristics, assessment and verification of constancy of performance and marking.

2. Moreno Berto A. (2007): Ceramic tiles: above and beyond traditional applications. Journal of the European Ceramic Society, 27, 1607-1613. doi: 10.1016/j.jeurceramsoc.2006. 04.146
3. Centro Ceramico's statistical data, internal report (2015).

4. Esposito L., Tucci A., Rastelli E., Palmonari C., Selli S. (2002): Stain resistance of porcelain stoneware tiles. American Ceramic Society Bulletin, 81 (10), 38-42.

5. Esposito L., Tucci A. (2000): Porcelain stoneware tile surfaces. American Ceramic Society Bulletin, 79 (5), 59-63.

6. Tucci A., Esposito L., Malmusi L., Piccinini A. (2002): Wear resistance and stain resistance of porcelain stoneware tiles. Key Eng. Mater., 206-213, 1759-1762. doi: 10.4028/ www.scientific.net/KEM.264-268.1519

7. Esposito L., Tucci A., Naldi D. (2005): The reliability of polished porcelain stoneware tiles. Journal of the European Ceramic Society, 25, 1487-1498. doi: 10.1016/j. jeurceramsoc.2004.05.030

8. Dondi M., Ercolani G., Guarini G., Melandri C., Raimondo M., Rocha e Almendra E, Tenorio Cavalcante P.M. (2005) : The role of surface microstructure on the resistance to stain of porcelain stoneware tiles. Journal of the European Ceramic Society, 25, 357-365. doi: 10.1016/j.jeurceramsoc. 2004.01.017

9. Raimondo M., Zanelli C., Dondi M., Guarini G., Biasini V., Gardini D. (2006): Surface properties of porcelain stoneware tiles: the influence of different protective coatings. in: Proceedings of IX Qualicer, Castellon, Spain, pp. P.BC 325- P.BC 333.

10. Akarsu M., Burunkaya E., Tunalı A., Selli N. T., Arpaç E. (2014): Enhancement of hybrid sol-gel coating and industrial application on polished porcelain stoneware tiles and investigation of the performance. Ceramics International, 40, 6533-6540. Doi: 10.1016/j.ceramint. 201.11.106

11. Miorin E., Pagura C., Battagliarin M., Fabrizio M., Guglielmi M., Miselli P. (2003): Stain-resistant sol-gel silica coatings on stoneware tile. American Ceramic Society Bulletin, 82 (3), 52-57.

12. EN 15802:2010. Conservation of cultural property. Test methods. Determination of static contact angle.

13. ISO 10545-14: 2015. Ceramic tiles, determination of resistance to stains.

14. ISO 10545-13: 2015. Ceramic tiles, determination of chemical resistance.

15. ISO 4287:1997. Geometrical Product Specifications (GPS) - Surface texture: Profile method -Terms, definitions and surface texture parameters.

16. EN 623-4: 2004. Advanced technical ceramics. Monolithic ceramics. General and textural properties. Determination of surface roughness. 